ARTICLE

https://doi.org/10.1038/s41467-019-11031-0

\title{
Neuroepigenetic signatures of age and sex in the living human brain
}

Tonya M. Gilbert1,4, Nicole R. Zürcher (10 1,4, Mary C. Catanese ${ }^{1}$, Chieh-En J. Tseng (1) 1, Maria A. Di Biase ${ }^{2}$, Amanda E. Lyall (1) 2,3, Baileigh G. Hightower ${ }^{1}$, Anjali J. Parmar ${ }^{1}$, Anisha Bhanot ${ }^{1}$, Christine J. Wu (1) ${ }^{1}$, Matthew L. Hibert ${ }^{1}$, Minhae Kim (D) 1, Umar Mahmood', Steven M. Stufflebeam (1D ${ }^{1}$, Frederick A. Schroeder ${ }^{1}$, Changning Wang1 ${ }^{1}$ Joshua L. Roffman ${ }^{1,3}$, Daphne J. Holt ${ }^{1,3}$, Douglas N. Greve1, Ofer Pasternak², Marek Kubicki2,3, Hsiao-Ying Wey ${ }^{1} \&$ Jacob M. Hooker ${ }^{1}$

Age- and sex-related alterations in gene transcription have been demonstrated, however the underlying mechanisms are unresolved. Neuroepigenetic pathways regulate gene transcription in the brain. Here, we measure in vivo expression of the epigenetic enzymes, histone deacetylases (HDACs), across healthy human aging and between sexes using $\left[{ }^{11} \mathrm{C}\right]$ Martinostat positron emission tomography (PET) neuroimaging $(n=41)$. Relative HDAC expression increases with age in cerebral white matter, and correlates with age-associated disruptions in white matter microstructure. A post mortem study confirmed that HDAC1 and HDAC2 paralogs are elevated in white matter tissue from elderly donors. There are also sexspecific in vivo HDAC expression differences in brain regions associated with emotion and memory, including the amygdala and hippocampus. Hippocampus and white matter HDAC expression negatively correlates with emotion regulation skills $(n=23)$. Age and sex are associated with HDAC expression in vivo, which could drive age- and sex-related transcriptional changes and impact human behavior.

\footnotetext{
${ }^{1}$ Athinoula A. Martinos Center for Biomedical Imaging, Department of Radiology, Massachusetts General Hospital, Harvard Medical School, Charlestown, MA 02129, USA. 2 Psychiatry Neuroimaging Laboratory, Departments of Psychiatry and Radiology, Brigham and Women's Hospital, Harvard Medical School, Boston, MA 02215, USA. ${ }^{3}$ Department of Psychiatry, Massachusetts General Hospital, Harvard Medical School, Boston, MA 02114, USA. ${ }^{4}$ These authors contributed equally: Tonya M. Gilbert, Nicole R. Zürcher. Correspondence and requests for materials should be addressed to J.M.H. (email: hooker@nmr.mgh. harvard.edu)
} 
A ge and sex represent two of the major biological variables that influence human health and disease. Aging is the main risk factor for neurodegenerative disease $\mathrm{e}^{1,2}$, and is associated with progressive deterioration of brain structure and function $^{3}$. Sex differences impact the incidence, severity, and treatment response in neurological and psychiatric disorders ${ }^{4,5}$. While age- and sex-related alterations in gene transcription have been demonstrated ${ }^{6-9}$, the underlying molecular mechanisms are unresolved. Genetic background alone cannot fully explain the relationship between these variables and changes in gene transcription. Epigenetic mechanisms, which integrate environmental and genetic factors, have a profound impact upon gene regulation that when altered, may drive disease risk ${ }^{10,11}$.

Dysregulation of histone deacetylases (HDACs), a family of epigenetic enzymes, could tip the balance from healthy to pathological aging. Hypoacetylation of specific histone marks is linked to age-related neurodegenerative diseases, as demonstrated by preclinical and post mortem studies ${ }^{12,13}$. In rodent models, HDAC expression ${ }^{14,15}$ and enzymatic activity ${ }^{16}$ increase with age in the hippocampus, leading to reductions in processes including histone acetylation ${ }^{17-19}$, transcription of synaptic plasticity genes $^{19-21}$, and memory formation ${ }^{19,21}$. HDAC inhibition alleviates age-related phenotypes ${ }^{12,17-19}$, such as deficiencies in gene transcription and cognitive performance that are present in human aging6,22. In the young adult human brain, HDAC expression is lowest in the white matter ${ }^{23}$. The effects of altered HDACs across lifespan are not fully understood. Interestingly, the efficiency of oligodendrocyte-mediated remyelination decreases with age in rodents, due to altered recruitment of HDACs at the promoter regions of myelin-associated genes ${ }^{24}$. Furthermore, HDAC inhibition reduces excitotoxicity ${ }^{25}$ and is protective against white matter injury in rodent disease models 26,27 .

Sexually dimorphic regulation of gene transcription by HDACs may contribute to differences in the prevalence of neurological and neuropsychiatric disorders between females and males ${ }^{4}$. For example, sex-specific HDAC expression patterns could impart susceptibility to memory-related disorders, supported by evidence from rodents of transient hypoacetylation in the hippocampus of females compared to males during early development ${ }^{28}$ and the positive relationship between histone acetylation and estrogeninduced memory enhancement in females ${ }^{29}$. Further, HDACs modulate and respond to sex steroid hormones ${ }^{29}$, influence sexual behavior ${ }^{30}$, and alter socio-emotional circuits in the limbic system $^{31}$. Moreover, HDACs play a fundamental role in male sex determination $^{32}$, female X-chromosome inactivation ${ }^{33}$, and genomic imprinting ${ }^{34}$.

Although HDACs are implicated in age- and sex-related vulnerability to disease through preclinical and post mortem studies, differences exist between these models and living humans at both molecular and systems levels. In humans, non-invasive measurement of in vivo HDAC expression is essential to investigate the contribution of neuroepigenetic mechanisms to age- and sexrelated alterations in gene transcription. Using $\left[{ }^{11} \mathrm{C}\right]$ Martinostat ${ }^{23,35-38}$, an HDAC positron emission tomography (PET) radiotracer, we previously mapped HDAC expression in healthy young-adults ${ }^{23}$. Here, we measure relative $\left.{ }^{11} \mathrm{C}\right]$ Martinostat uptake in the brains of healthy individuals across the adult lifespan, and compare HDAC expression patterns between females and males. We then apply simultaneously acquired diffusion magnetic resonance (MR) imaging and cognitive performance testing to relate HDAC expression levels with alterations in white matter microstructure and human socio-emotional behavior, respectively. We find that relative HDAC expression increases with age in cerebral white matter and correlates with alterations in white matter microstructure. We also identify sexspecific HDAC expression differences in brain regions associated with socio-emotional processes, including the amygdala and hippocampus. We provide preliminary evidence that HDAC expression in both the hippocampus and white matter negatively correlates with emotion regulation skills. We propose that HDACs are strongly associated with the major biological variables of age and sex, and may represent potential targets to modify age- and sex-related pathophysiology.

\section{Results}

Study design. To characterize HDAC expression patterns across the adult lifespan and between females and males, healthy adults ( $n=41$ subjects, mean age \pm standard deviation $=41 \pm 17$ years, 18-79-years-old, 20 females, 21 males) underwent simultaneous MR-PET with $\left[{ }^{11} \mathrm{C}\right]$ Martinostat, a radiotracer selective for HDAC paralogs $1,2,3$, and putatively $6^{23,35-38}$. [ $\left.{ }^{11} \mathrm{C}\right]$ Martinostat was previously found to engage HDAC1, HDAC2, and HDAC3 in both in vitro assays using recombinant proteins ${ }^{35}$ and ex vivo assays using human brain tissue ${ }^{23}$, while HDAC6 was engaged only in in vitro assays 23,35 .

[ $\left.{ }^{11} \mathrm{C}\right]$ Martinostat SUVR is a surrogate measure for $V_{\mathrm{T}}$. Given the risks and invasiveness of arterial blood sampling, we first determined that standard uptake value (SUV) normalized to whole brain mean (standard uptake value ratio, SUVR) from 60 to $90 \mathrm{~min}$ post radiotracer injection is an appropriate surrogate measure for the distribution volume $\left(V_{\mathrm{T}}\right)$ in a subset of healthy young and old adults. Regional SUVR were strongly correlated with $V_{\mathrm{T}}$ values derived from a two-tissue compartmental model (2TCM), using metabolite-corrected arterial plasma as an input function (all Pearson's $r \geq 0.90$, all $P<0.0001$, Supplementary Fig. 1). SUVR was therefore used as the primary outcome measure to determine relative changes in $\left[{ }^{11} \mathrm{C}\right]$ Martinostat uptake $^{38-40}$

SUVR increases with age in the white matter. First, voxel-wise analysis correlating $\left[{ }^{11} \mathrm{C}\right]$ Martinostat SUVR with age was performed while controlling for sex and brain volumes ${ }^{41}$ (grey matter, white matter, and cerebrospinal fluid volumes normalized by estimated total intracranial volume) $\left(P_{F W E}<0.05\right.$, Fig. 1, Supplementary Note 1, Supplementary Fig. 2). We observed a significant increase in HDAC expression with age in the cerebral white matter, but not the corpus callosum (Fig. 1). Analysis in native space (i.e. individual anatomically defined regions for every subject) supported these results (Supplementary Fig. 3a). The native space cerebral white matter masks were eroded in order to assess SUVR changes in deep white matter, where physical contact with the ventricles (a major source of partial volume effects) was limited. SUVR remained strongly correlated with age in the eroded masks (Supplementary Fig. 3b). Partial volume correction was applied using two complementary approaches: (1) voxel-wise correlations of SUVR with age were restricted to white matter, controlled for sex and region-based voxel-wise (RBV) correction was applied (Supplementary Fig. 4a) and (2) SUVR was extracted from cerebral white matter in native space using the geometric transfer matrix (GTM) and correlated to age (Supplementary Fig. 4b). Partial volume corrected SUVR and age were correlated in the cerebral white matter, confirming our findings. Further, these results were not driven by body mass index (BMI) and were recapitulated using a secondary normalization method (Supplementary Notes 2 and 3, Supplementary Figs. 5-7). Interestingly, SUVR in the cerebral white matter increased with age only in mid to late adulthood, as demonstrated by correlation analysis split at the median age of 35 years. A positive correlation was found in subjects older than 35 years (Spearman's $r=0.44, P<0.05$ ), whereas no correlation was observed in subjects equal or younger 


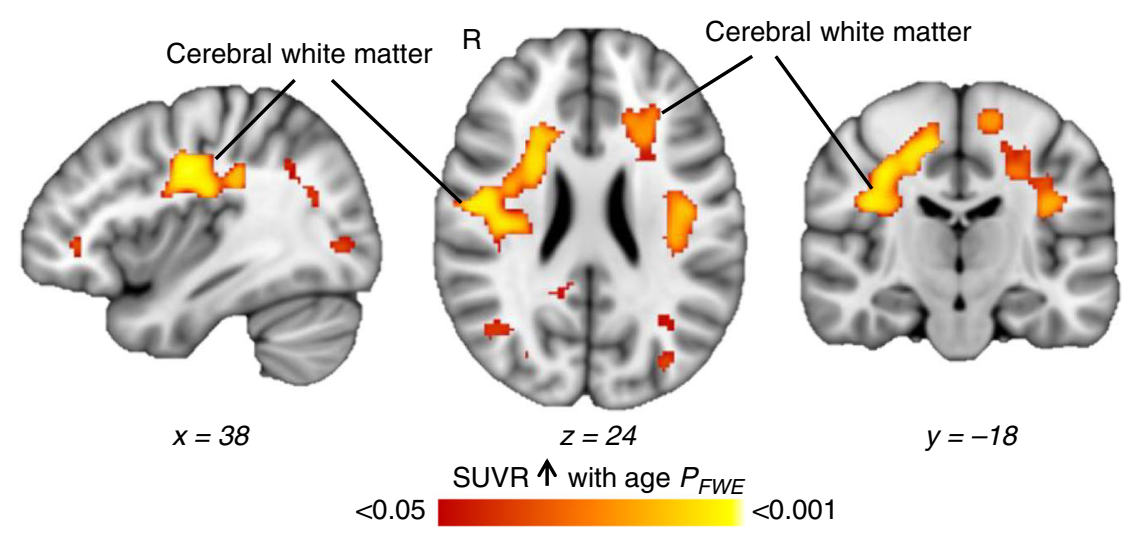

Fig. $1\left[{ }^{11} \mathrm{C}\right]$ Martinostat uptake increases with age in the white matter. Voxel-wise correlations of SUVR with age, controlled for sex, total grey matter volume/intracranial volume, white matter volume/intracranial volume, and cerebrospinal fluid volume/intracranial volume $(n=41)$. Statistical maps were overlaid onto the MNI $1 \mathrm{~mm}$ template in radiological orientation (family wise error rate (FWE) corrected for multiple comparisons, $P_{F W E}<0.05$; nonparametric permutation testing $n=10,000$ permutations). Red-yellow represents regions significantly increased with age

than 35 years (Spearman's $r=-0.082, P=0.73$, Supplementary Fig. 8).

Second, based on previous ex vivo studies that reported elevated HDAC expression and deficient histone acetylation in the hippocampus of aged rodents ${ }^{14,17-19,42}$, the hippocampus was selected for a priori region of interest (ROI) analysis and GTM partial volume correction was applied. However, contrary to rodent studies, HDAC expression and age were not strongly correlated in the human hippocampus, despite the presence of a trend (Spearman's $r=0.29, P=0.062$, Supplementary Fig. 9).

SUVR correlates with alterations in white matter structure. Taking advantage of simultaneous MR-PET methodology, we sought to determine whether age-associated increases in HDAC expression are linked with changes in white matter microstructure. We analyzed generalized fractional anisotropy ( $\mathrm{gFA}$ ), a diffusion metric similar to fractional anisotropy (FA), which represents white matter microstructural organization. Analysis of gFA is believed to be less sensitive to measurement errors that result from crossing fibers compared to $\mathrm{FA}^{43-45}$. First, ROI analysis guided by our PET finding (Fig. 1) was performed to determine the relationship between $\left[{ }^{11} \mathrm{C}\right]$ Martinostat SUVR and gFA. SUVR and gFA values were extracted from the post-hoc PET ROI where SUVR increased with age, restricted to white matter areas. See Fig. 2a for ROI description. In this ROI, SUVR negatively correlated with gFA (Spearman's $r=-0.37, P=0.018$, Fig. 2a). Next, voxel-wise analysis correlating gFA with age while controlling for sex $\left(P_{F W E}<0.05\right.$, Fig. 2 b) was performed across the whole brain to determine co-localization between alterations in white matter microstructure and increases in HDAC expression. Age-related gFA decreases showed extensive spatial overlap with age-related SUVR increases (Fig. 2b).

HDAC1 and HDAC2 increases in elderly white matter tissue. To investigate age-related increases of $\left[{ }^{11} \mathrm{C}\right]$ Martinostat uptake at the molecular level, post mortem human brain tissue was obtained from older donors $(n=9$, mean age \pm standard deviation $=85 \pm 8$ years $)$ and younger donors $(n=9$, mean age $=18 \pm$ 1 year) matched for sex ( $n=4$ females and $n=5$ males in each group). Periventricular white matter (PVM) was chosen because $\left[{ }^{11} \mathrm{C}\right]$ Martinostat SUVR increased with age in PVM (Fig. 1) and PVM lesions are observed in aged individuals ${ }^{46}$. Corpus callosum (CC) was used as a negative control, because we did not observe SUVR alterations in this white matter region (Fig. 1). Protein expression of HDACs (paralogs 1,2,3, and 6) relative to glyceraldehyde-3-phosphate dehydrogenase (GAPDH) were assessed by western blotting. Expression of HDAC1 and HDAC2, paralogs previously implicated in oligodendrocyte differentiation and myelination ${ }^{24,47-49}$, were significantly higher in older donors compared to younger donors in the PVM, whereas HDAC3 and HDAC6 were not different between groups (two-tailed unpaired $t$-test, false discovery rate (FDR) corrected for multiple comparisons; HDAC1: $P=0.022$ and $P_{F D R}=0.031$, HDAC2: $P=0.018$ and $P_{F D R}=0.29$, HDAC3: $P=0.62$ and $P_{F D R}=0.81$, HDAC6: $P$ and $P_{F D R}>0.99$, Fig. 3a,b, Supplementary Fig. 10). In the CC, none of the tested HDAC paralogs were significantly different between groups (two-tailed unpaired $t$-test, FDR corrected for multiple comparisons; HDAC1: $P=0.30$ and $P_{F D R}=0.27$, HDAC2: $P=0.15$ and $P_{F D R}=0.28$, HDAC3: $P=0.41$ and $P_{F D R}=0.35$, HDAC6: $P=0.73$ and $P_{F D R}=0.70$, Fig. $3 c$, Supplementary Fig. 11). Our post mortem HDAC expression data suggest that the observed increase in $\left[{ }^{11} \mathrm{C}\right]$ Martinostat uptake in the PVM is due to an increase in HDAC1 and HDAC2.

SUVR shows sex-specific regional distribution. HDAC expression was compared between female $(n=20)$ and male $(n=21)$ subjects (Table 1, Supplementary Fig. 12a). Female and male subjects did not differ in age. Voxel-wise analysis of $\left[{ }^{11} \mathrm{C}\right] \mathrm{Mar}-$ tinostat SUVR was performed between groups controlling for age and brain volumes ( $Z$ score $>2.3, P_{\text {cluster }}<0.05$, Fig. 4 , Supplementary Note 1, Supplementary Fig. 12b). SUVR was higher in females compared to males in the frontal medial cortex, amygdala, hippocampus, parahippocampal gyrus, and thalamus (Fig. 4). SUVR was lower in females compared to males in cerebellar white matter (Fig. 4). These results were recapitulated using a secondary normalization method (Supplementary Note 3, Supplementary Fig. 13).

SUVR may correlate with emotion regulation skills. To investigate the potential impact of changes in $\left[{ }^{11} \mathrm{C}\right]$ Martinostat uptake on human behavior, exploratory correlation analysis was performed between SUVR and cognition in a subset of subjects $(n=23$, mean age \pm standard deviation $=50 \pm 17$ years, $23-79$ years-old, 12 females, 11 males) who underwent the Mayer-Salovey-Caruso Emotional Intelligence Test (MSCEIT) ${ }^{50}$. The MSCEIT is a socio-cognitive test that measures emotion regulation skills and social judgement. In a previous study, we determined that $\left[{ }^{11} \mathrm{C}\right]$ Martinostat SUVR in the dorsolateral prefrontal cortex was associated with the MSCEIT score, across subjects with schizophrenia and healthy controls ${ }^{38}$. Therefore, the 
a

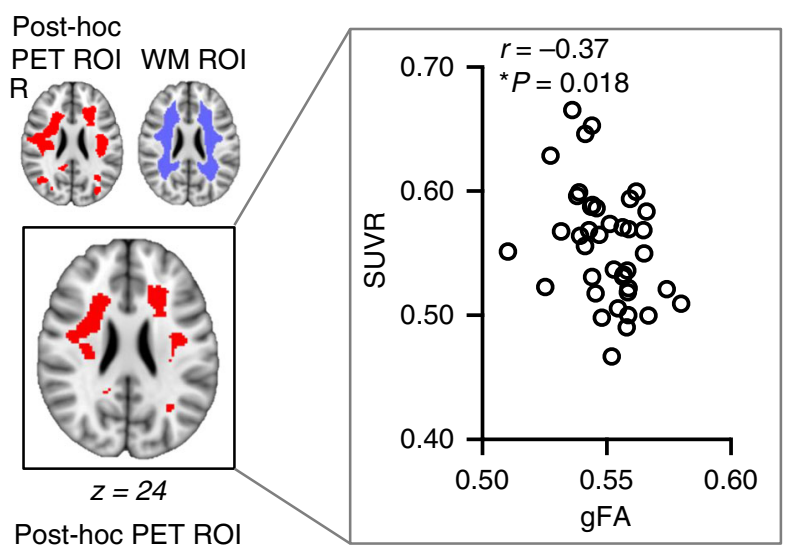

constrained to WM

b

SUVR
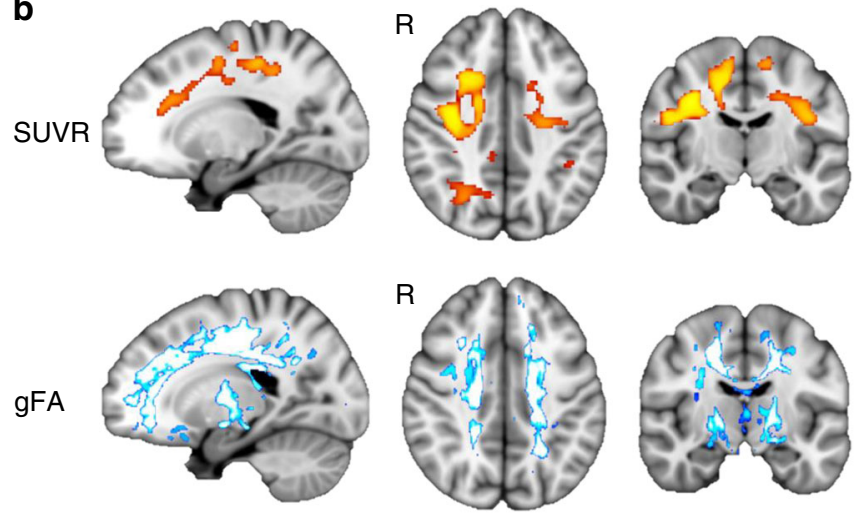

$x=-18$

$z=38$

$y=-12$

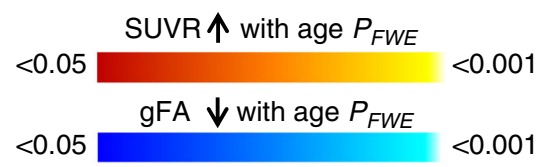

Fig. $2\left[{ }^{11} \mathrm{C}\right]$ Martinostat uptake correlates and co-localizes with alterations in white matter microstructure. a (Left) A post-hoc PET ROI was created from a binary mask of the entire statistical map in Fig. 1 (red, depicting regions where SUVR increased with age), and was constrained to white matter. ROIs are overlaid onto the MNI $1 \mathrm{~mm}$ template in radiological orientation. (Right) Spearman correlation analysis between SUVR and gFA values extracted from the post-hoc PET ROI constrained to white matter $(n=40)$. $\mathbf{b}$ Voxel-wise correlations of (Top) SUVR increases with age, controlled for sex, total grey matter volume/intracranial volume, white matter volume/intracranial volume, and cerebrospinal fluid volume/ intracranial volume $(n=41)$ and (Bottom) gFA decreases with age, controlled for sex $(n=40)$. Statistical maps were overlaid onto the MNI 1 $\mathrm{mm}$ template in radiological orientation $\left(P_{F W E}<0.05\right.$; non-parametric permutation testing $n=10,000$ permutations). Red-yellow represents regions where SUVR significantly increased with age and blue-light blue represents regions where gFA significantly decreased with age

MSCEIT score was chosen as the exploratory cognitive metric in this study. Preliminary voxel-wise analysis correlating SUVR with MSCEIT scores was performed while controlling for age, sex and brain volumes $\left(Z\right.$ score $>2.3, P_{\text {cluster }}<0.05$, Supplementary Fig. 14a). We observed a significant decrease in HDAC expression with MSCEIT scores in the cerebral white matter, specifically the inferior fronto-occipital fasciculus (IFOF) and the inferior longitudinal fasciculus (ILF) tracts, and the hippocampus (Supplementary Fig. 14a). Both the IFOF and ILF have previously been linked to multiple aspects of social cognition, including face perception and theory of mind ${ }^{51,52}$. In the hippocampus, HDACs negatively regulate the transcription of synaptic plasticity genes $^{19-21}$, memory formation ${ }^{14,21}$ and mood-related social behaviors $^{53,54}$ in rodents. Therefore, the hippocampus was selected for preliminary a priori ROI analysis and GTM partial volume correction was applied. In the human hippocampus, SUVR negatively correlated with MSCEIT scores (Spearman's $r=-0.43, P=0.043$, Supplementary Fig. 14b).

\section{Discussion}

We discovered that HDACs, an epigenetic enzyme family with critical importance to neural processes, show age-related and sexspecific in vivo expression differences in the human brain. Previous studies relied on ex vivo measurements in preclinical rodent models, which have a shorter lifespan and more rapid sexual development compared to humans. Our work has the unique advantage of non-invasive assessment of HDACs throughout the living human brain.

Aging is the strongest risk factor for neurodegenerative diseases, such as Alzheimer's disease and Parkinson's disease ${ }^{2,55}$, and it contributes to pathophysiology in ways that are unresolved. We reveal through in vivo imaging, and validate through post mortem biochemistry, that HDAC1 and HDAC2 increase by as much as $30 \%$ in periventricular white matter across the adult lifespan. Interestingly, HDAC expression negatively correlates with ageassociated alterations in white matter microstructure (gFA). Preliminary evidence also suggests that HDAC expression may negatively correlate with emotion regulation skills in the IFOF and ILF tracts, white matter areas linked to face processing, empathy, emotion recognition, and theory of mind ${ }^{51,52}$. We propose that these relationships could represent a mechanistic link between aging and perhaps cognitive performance, with neuroepigenetic regulation of genes that control white matter microstructural organization. More specifically, myelination may be implicated as HDAC1 and HDAC2 play key roles in remyelination in both the central and peripheral nervous systems ${ }^{24,26,47,48,56}$. Recent epigenomic analyses in post mortem brain tissues have identified distinct changes in histone acetylation and downstream gene transcription in neurodegenerative disease $\mathrm{e}^{13,57,58}$, with a redistribution of epigenetic signatures observed between healthy aging and disease states ${ }^{13,57}$. Taken together with the increased HDAC2 expression observed in post mortem brain tissue from Alzheimer's disease patients ${ }^{20}$, it is possible that HDAC1/2 accumulation in white matter may link aging to neurodegenerative disease through transcriptional dysregulation. Having determined the localization and magnitude of the HDAC1/2 increase, we are now poised to identify the specific cell-types involved and determine the downstream consequences of increased HDACs in the aging brain.

We observed a trend for a positive correlation between age and in vivo HDAC expression in the human hippocampus, whereas previous rodent studies reported significant age-related changes of HDAC expression ${ }^{14,19}$. It is possible that age-related changes are focal in subfields of the hippocampus such as CA1 15,18,42,59, and are not detectable with our current methodology. We did observe novel sex-specific differences in HDAC expression throughout the limbic system, with higher HDAC expression in the subregions of the hippocampus, amygdala, and parahippocampus of females compared to males. Furthermore, SUVR in the hippocampus may negatively correlate with emotion regulation skills. Sexually dimorphic expression of HDAC could be relevant to the higher prevalence of neuropsychiatric disorders and neurodegenerative diseases such as major depression, generalized anxiety disorder, and Alzheimer's disease, in females. Structural, functional, and neurochemical sexual dimorphisms including differences in dopamine, GABA, and serotonin 


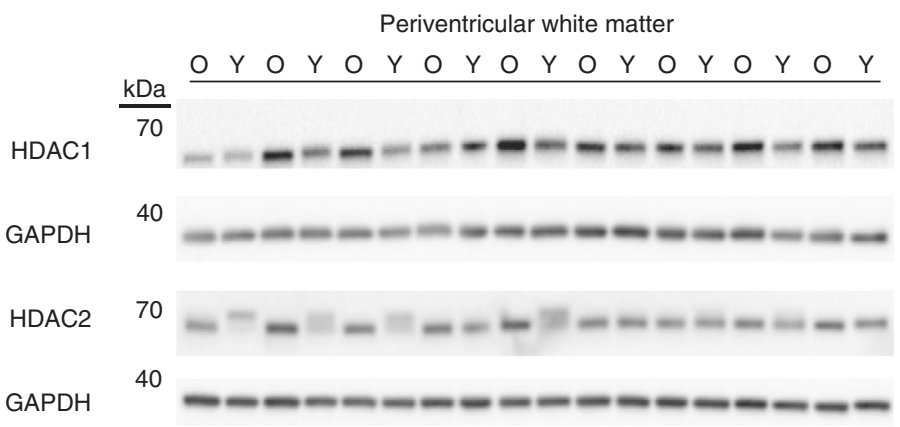

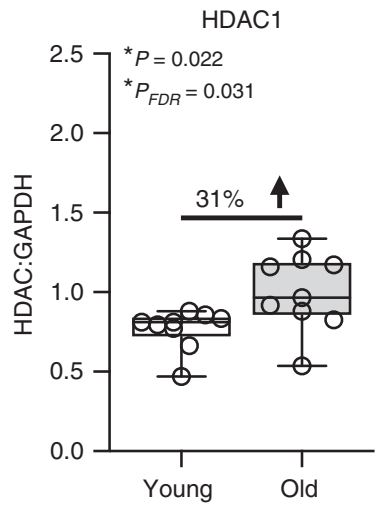

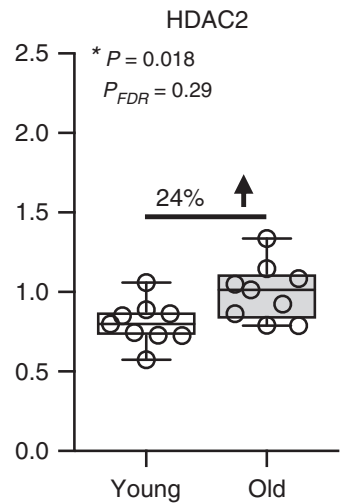

b

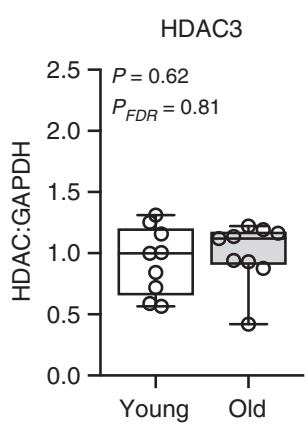

C

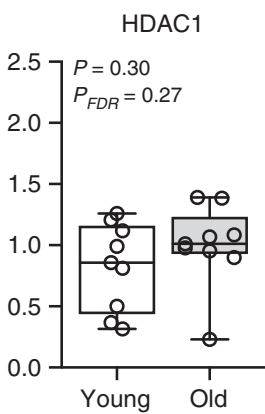

HDAC6

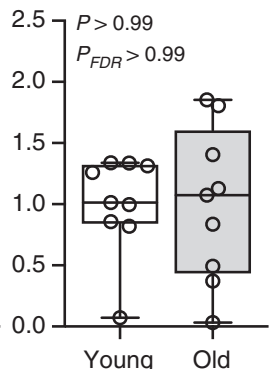

Corpus Callosum (control region)
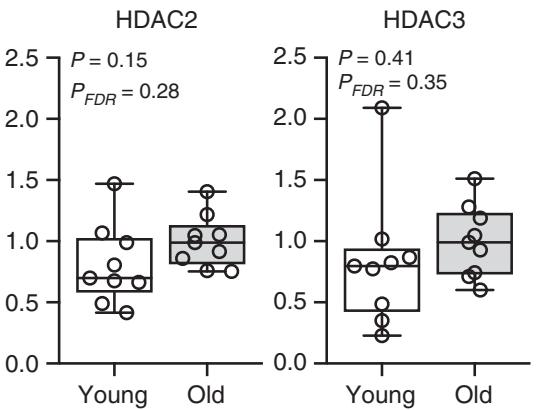

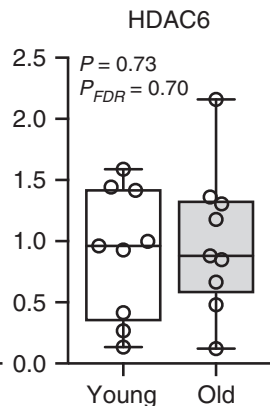

Fig. 3 HDAC1 and HDAC2 expression is increased in the white matter of elderly donors. Whole-cell lysates were prepared from post mortem periventricular white matter and corpus callosum tissues. Protein expression of HDAC paralogs (1, 2, 3, and 6) were compared between younger donors $(n=9,16-21$ years -old) and older donors $(n=9,76-100$-years-old) by western blotting (two-tailed unpaired $t$-test; false discovery rate (FDR) corrected for multiple comparisons). For each paralog, immunoreactive band intensity was normalized by GAPDH and scaled to a mean of 1 . Box plots display median, first quartile, third quartile, and range (min-max) from a, b periventricular white matter and c corpus callosum. See Supplementary Figs. 10 and 11 for western blot images

\section{Table 1 Subject demographic information}

\begin{tabular}{lll} 
Characteristic & Females & Males \\
\hline Number of subjects & 20 & 21 \\
Age range (years) & $18-74$ & $20-79$ \\
Age mean \pm SD (years) & $40.8 \pm 18.1$ & $40.7 \pm 16.9$ \\
Body mass index mean \pm SD & $25.0 \pm 4.0$ & $24.5 \pm 4.2$ \\
Injected dose mean \pm SD $(\mathrm{mCi})$ & $5.1 \pm 0.43$ & $4.8 \pm 0.65$ \\
Molar activity mean \pm SD $(\mathrm{mCi} / \mathrm{nmol})$ & $1.9 \pm 0.72$ & $1.9 \pm 0.73$ \\
\hline
\end{tabular}

neurotransmitter levels have been reported ${ }^{60}$. For example, in vivo expression of the serotonin receptor $\left(5-\mathrm{HTR}_{1 \mathrm{~A}}\right)$ is increased in the hippocampus and amygdala of females compared to males in humans ${ }^{61,62}$. Transcription of $5-H T R_{1 A}$ mRNA is regulated in part by $\mathrm{HDAC} 1 / 2^{63}$, which suggests that altered HDAC levels could affect neurotransmitter systems. Additionally, serotonin-dependent activation of the serotonin receptor $\left(5-\mathrm{HTR}_{2 \mathrm{~A}}\right)$ inhibits $H D A C 2$ promoter activity in human cells ${ }^{64}$, while $5-\mathrm{HTR}_{2 \mathrm{~A}}$ inhibition increases $\mathrm{Hdac2}$ transcription and negatively affects synaptic remodeling and cognitive behavior through NF- $\mathrm{kB}$ signaling in rodents ${ }^{65}$, which may have relevance to depression and other neuropsychiatric disorders. Indeed, HDAC inhibitors elicit anti-depressive effects and alter social behavior in rodent models of neuropsychiatric disorders ${ }^{54,64-68}$. Moreover, in the hippocampus, HDACs also regulate the transcription of genes critical for synaptic plasticity ${ }^{19-21}$ and HDAC inhibition rescues memory deficits in rodent models of neurodegeneration ${ }^{18,69,70}$.

Due to the pleiotropic effects of HDACs on gene transcription, small changes in HDAC expression could have a large impact on neural circuits. For example, genetic knockdown of Hdac2 expression by $25-30 \%$ increased transcription of memory-related genes and synaptic protein density, rescued long-term potentiation, and improved associative and spatial memory in an Alzheimer's disease rodent $\operatorname{model}^{20}$. Furthermore, selective inhibition of HDAC1/2 modulated the transcription of over 1600 genes in the rodent brain ${ }^{66}$. These data support the hypothesis that the age-related and sex-specific in vivo HDAC expression patterns we observe in humans may influence neural function. The HDAC signal detected in each brain region, and each context (i.e. age or sex differences) may potentially correspond to a different subset of HDACs engaged by $\left[{ }^{11} \mathrm{C}\right]$ Martinostat. Previous work has demonstrated that $\left[{ }^{11} \mathrm{C}\right]$ Martinostat binds $\mathrm{HDACl}$, HDAC2, HDAC3, and to a lesser extent HDAC6 ${ }^{23,35}$, thus we currently detect HDAC expression in aggregate rather than expression of individual paralogs. Therefore, as we translate paralog-selective HDAC imaging tools ${ }^{71}$ for human use, we will be better equipped to further interpret signal changes and relate each paralog to its respective phenotypes.

Our results suggest that in humans, age and sex are strongly associated with alterations in HDAC expression that may influence human behavior. As these biological variables contribute to disease risk, pharmacological agents such as HDAC inhibitors 

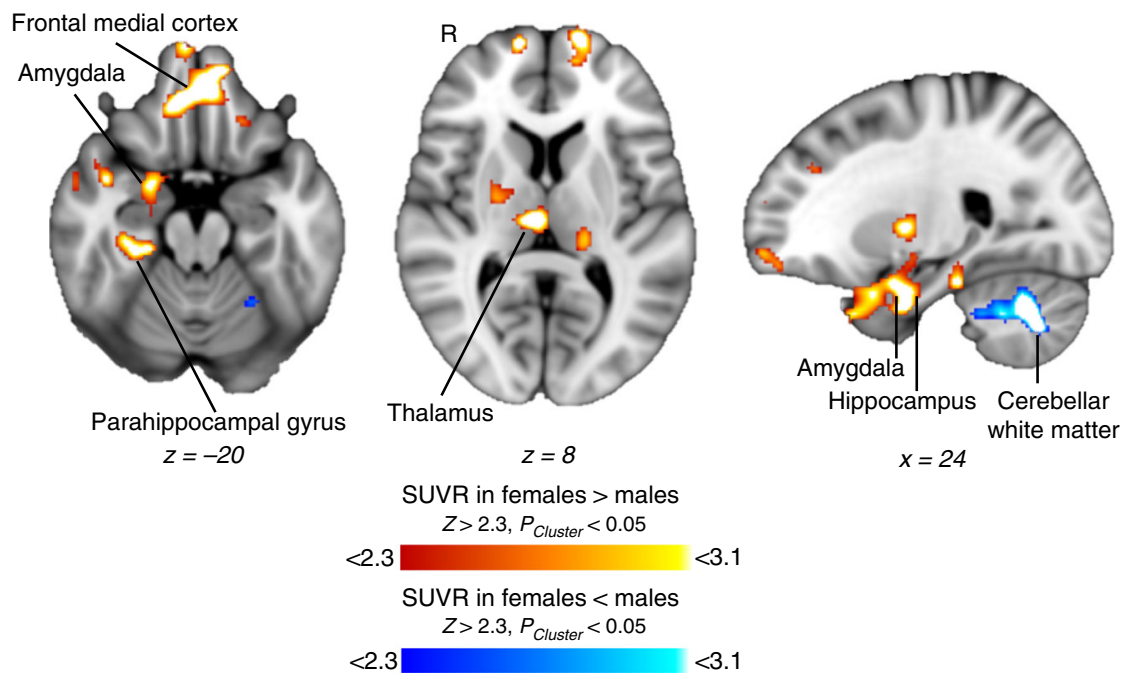

Fig. $4\left[{ }^{11} \mathrm{C}\right]$ Martinostat uptake shows sex-specific differences. Voxel-wise comparison of SUVR between females $(n=20)$ and males $(n=21)$, controlled for age, total grey matter volume/intracranial volume, white matter volume/intracranial volume and cerebrospinal fluid volume/intracranial volume. Statistical maps were overlaid onto the MNI $1 \mathrm{~mm}$ template in radiological orientation (two-tailed unpaired $t$-test; $Z>2.3, P_{\text {Cluster }}<0.05$ ). Red-yellow represents regions significantly increased in females compared to males and blue-light blue represents regions significantly decreased in females compared to males

may represent a potential treatment to modify or intervene in pathophysiology.

\section{Methods}

Study design. Our main research objective was to compare $\left[{ }^{11} \mathrm{C}\right]$ Martinostat brain uptake across age and between sex in healthy adult subjects, using MR-PET. SUV normalized to whole brain mean (SUVR) from 60-90 min post radiotracer injection was the primary endpoint assessed. The study was approved by the Partners HealthCare Institutional Review Board (IRB) and the Massachusetts General Hospital (MGH) Radioactive Drug Research Committee. All subjects provided written informed consent according to the Declaration of Helsinki. Imaging procedures were performed at the Athinoula A. Martinos Center for Biomedical Imaging. See Table 1 for demographic information. Subjects underwent a physical examination by a licensed physician or nurse practitioner. Medical and medication history were recorded. Illicit and psychotropic drug use was assessed by a urine drug screen. A serum pregnancy test was performed for female subjects to ensure no pregnancy at the time of the scan. Forty-two subjects were scanned, but one subject was excluded from primary analyses for a dental work-related artifact. Diffusion images were available to assess white matter microstructure in forty subjects. MSCEIT ${ }^{50}$ scores were available to assess cognitive performance in twenty-three subjects; scores were age- and sex-corrected. We furthered our findings through biochemical analysis of post mortem human brain tissue from eighteen donors, to measure protein expression of HDAC paralogs. Imaging and biochemical studies were not blinded, and no outliers were excluded.

Subject inclusion/exclusion criteria. Subjects were healthy as determined by a licensed physician/nurse practitioner. Subjects had no history of psychiatric or major physical illness (including but not limited to depression, schizophrenia, mild cognitive impairment, diabetes mellitus, or head trauma). Subjects had no present substance abuse. Subjects who were taking psychotropic medications, or using illicit drugs or marijuana, were excluded. Subjects were excluded if they met any MR-PET safety contraindications, including pregnancy or breastfeeding.

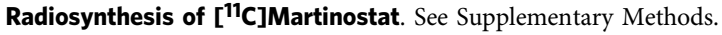

MR-PET data acquisition. See Supplementary Methods.

MR data analysis. See Supplementary Methods.

Primary PET image analysis. Primary analysis was performed in 41 subjects (18-79-years-old, 20 females, 21 males). Motion correction was applied with the MCFLIRT tool ${ }^{72}$ in FSL version 5.0.7 $7^{73}$. Motion-corrected SUV frames from 60-90 min were averaged to create a mean SUV image for each subject. SUV images were registered to the subject's MEMPRAGE using mri_coreg from FreeSurfer 6.0, skullstripped, and resampled to 2-mm isotropic voxel size. The subject's MEMPRAGE was registered to MNI space using linear FLIRT ${ }^{72}$ and nonlinear FNIRT $^{74}$ algorithms in FSL. The registration matrix was then applied to move SUV images into MNI space. SUV images in native space and MNI space were (1) intensity normalized to whole brain mean as SUVR ${ }^{38-40}$ and (2) intensity normalized to mean pons SUV as SUVR Pons, $_{\text {, }}$ using individual native space pons masks generated by FreeSurfer's automated brainstem segmentation ${ }^{75}$. SUVR and SUVR Pons $_{\text {images }}$ were spatially smoothed $8 \mathrm{~mm}$ full width at half maximum (FWHM). Whole brain voxel-wise analysis was performed with smoothed SUVR and SUVR Pons $_{\text {images in }}$ MNI space using FSL's randomise ${ }^{76}$ and FEAT $^{77}$. Region of interest (ROI) analysis was performed with unsmoothed SUVR images in native space using masks for the hippocampus and cerebral white matter generated by FreeSurfer's automated parcellation and segmentation ${ }^{78}$. ROI analysis was also performed with unsmoothed SUVR images in native space using eroded cerebral white matter masks.

Partial volume correction. Partial volume correction was applied using RBV analysis and ROI based GTM, both available in PETSurfer ${ }^{79}$

Quantitative analysis using kinetic modeling. An arterial line (A-line) was placed in a subset of subjects ( $n=7,22-53$-years-old, two females, five males) in order to perform full quantitative analysis to determine distribution volume $\left(V_{\mathrm{T}}\right)$. The A-line was placed in the radial artery by a licensed anesthesiologist for arterial blood sampling to determine plasma radioactivity and radioactive metabolites. Blood samples were drawn by an experienced nurse practitioner. Arterial plasma and metabolite analyses were performed. PET images were reconstructed with the following scheme $(10 \mathrm{~s} \times 8,20 \mathrm{~s} \times 3,30 \mathrm{~s} \times 2,60 \mathrm{~s} \times 1,120 \mathrm{~s} \times 1,180 \mathrm{~s} \times 1,300 \mathrm{~s} \times 8$, and $600 \mathrm{~s} \times 4)$ and reconstructed in units of both bqml and SUV. Kinetic modeling was executed with PMOD version 3.4 (PMOD Technologies Ltd., Zurich, Switzerland). A 2 TCM was applied to the regional time-activity curves (TACs) extracted from volumes of interest (VOIs), and the metabolite-corrected arterial plasma was used as the input function to derive $V_{\mathrm{T}}$. VOIs were defined according to the Automated Anatomical Labeling (AAL) human brain atlas distributed with $\mathrm{PMOD}^{80}$. As done previously, we included fourteen VOIs with bilateral VOIs merged together ${ }^{23}$. The VOIs included composite VOIs for the frontal, parietal, temporal and occipital lobes, as well as VOIs for the insula, cingulate, caudate, putamen, pallidum, thalamus, hippocampus, amygdala, cerebellum, and white matter. SUV from 60 to $90 \mathrm{~min}$ post radiotracer injection was normalized to whole brain mean (SUVR). SUV from 60 to 90 min post radiotracer injection was also normalized to mean pons SUV (SUVR Pons $\left._{\text {) }}\right) V_{\text {T }}$, SUVR, and SUVR Pons $_{\text {images were }}$ spatially smoothed with an $8 \mathrm{~mm}$ FWHM gaussian kernel. Pearson correlation was then conducted between SUVR (or SUVR Pons) and $V_{\mathrm{T}}$ values for the fourteen VOIs, for each subject.

Post mortem tissue analysis. Human brain tissue was obtained from the National Institutes of Health (NIH) NeuroBioBank, specifically the Harvard Brain Tissue Resource Center and the University of Maryland Brain and Tissue Bank. Informed consent was obtained from next of kin for all donors. Donor brains had a neuropathology diagnosis of normal. Periventricular white matter or corpus callosum was homogenized in lysis buffer containing PBS, $0.15 \%$ NP-40, and a protease 
inhibitor cocktail (Roche \#11836170001) at $50 \mathrm{mg} / \mathrm{ml}$. Homogenate was sonicated at $50 \%$ power (Fisher Scientific Model \#CL-18) on ice for thirty pulses of $1 \mathrm{~s}$ duration followed by $2 \mathrm{~s}$ of rest. Tissue lysate was plunged through a 23 -gauge syringe 10 times, incubated with rotation at $4{ }^{\circ} \mathrm{C}$ for $20 \mathrm{~min}$, and centrifuged $18,000 \times g$ at $4{ }^{\circ} \mathrm{C}$ for $20 \mathrm{~min}$. Supernatant was collected and total protein concentration was measured using a bicinchoninic acid (BCA) protein assay (Pierce \#23227). Lysate (12 $\mu \mathrm{g}$ per sample) was separated with Criterion Stain-Free 4-20\% gels (Biorad \#567-8095) and transferred to PVDF membrane (Biorad \#162-0264). Membranes were blocked in 5\% milk (Biorad \#170-6404) prepared in tris buffered saline with $0.1 \%$ Tween 20 (TBST) overnight at $4{ }^{\circ} \mathrm{C}$. Remaining procedures were performed at room temperature. Membranes were washed in TBST and incubated with primary antibodies in TBST containing $1 \%$ milk for 90 min (HDAC1: Thermo Fisher \#PA1-860 at 1:6000, HDAC2: Abcam \#ab124974 at 1:5000, HDAC3: Abcam \#ab32369 at 1:2500, HDAC6: Santa Cruz \#sc11420 at 1:6000, GAPDH: Abcam ab8245 at 1:2000000). Membranes were washed in TBST and incubated with secondary antibodies in TBST containing $1 \%$ milk for 60 min (anti-rabbit-HRP: Cell signaling \#7074S at 1:5000, anti-mouse HRP: Cell Signaling \#7076S at 1:5000). Membranes were washed in TBST, developed with ECL reagent (GE Healthcare \#RPN2232), and visualized with a Chemidoc XRS imager (Biorad \#170-8265). For analysis, western blot images were converted from Image lab 5.2.1 files (.scn) to 600 dpi and saved as Tif files. The images were analyzed in Image J after conversion to 8-bit, subtraction of background with a 50-pixel rolling ball radius, and inversion. Mean band intensity was quantified using the measurement tool. See Supplementary Figs10. and 11 for uncropped western blot images.

Statistical analysis. FSL was used for voxel-wise analyses using FEAT or randomise. For all other analyses, GraphPad Prism version 8.01 was used.

For voxel-wise comparisons (Figs. 1 and 2, Supplementary Figs. 4a, 7a, b, and 13b), FSL's randomise was used with threshold-free cluster enhancement (TFCE) and 10,000 permutations, with family wise error rate (FWE) to correct for multiple comparisons $\left(P_{F W E}<0.05\right)$. For voxel-wise comparisons (Fig. 4, Supplementary Figs. 13a and 14a), FSL's FEAT with ordinary least squares (OLS) mixed-effects modeling was used with $Z$ score $>2.3$ and $P_{\text {cluster }}<0.05$. Voxel-wise analyses were conducted with the following regressors: (1) age vs. SUVR: sex, total grey matter volume/intracranial volume (ICV), white matter volume/ICV and cerebrospinal fluid volume/ICV; (2) age vs. SUVR (within WM using region-based voxel-wise partial volume correction), age vs. SUVR $\mathrm{Pons}_{\text {, }}$ as well as age vs. gFA: sex; (3) sex difference in SUVR: age, total grey matter volume/ICV, white matter volume/ICV and cerebrospinal fluid volume/ICV; (4) sex difference in SUVR Pons: $_{\text {age; (5) }}$ MSCEIT T-scores vs. SUVR: age, sex, total grey matter volume/ICV, white matter volume/ICV and cerebrospinal fluid volume/ICV.

For ROI analyses, (1) Pearson correlations to relate $\left(V_{\mathrm{T}}\right.$ to SUVR or SUVR $\mathrm{Pons}_{\text {; }}$ (2) Spearman rank-order correlations to relate age with SUVR or SUVR Pons, $_{\text {, age }}$ with brain volumes, SUVR with BMI, SUV in the whole brain with SUV in the pons, gFA with SUVR, and MSCEIT T-scores with SUVR; (3) two-tailed unpaired Mann-Whitney $U$-test to assess differences in age or brain volumes between females and males.

For biochemical analyses, two-tailed unpaired $t$-tests comparing individual HDAC paralog expression between older and younger donor tissues with FDR correction for multiple comparisons.

Reporting summary. Further information on research design is available in the Nature Research Reporting Summary linked to this article.

\section{Data availability}

The data that support these findings are available from the corresponding author, J.M.H., upon reasonable request. Human subject data will be deidentified to protect confidentiality.

\section{Code availability}

Custom codes for MR and PET data processing are available from the corresponding author, J.M.H., upon reasonable request.

Received: 23 April 2019 Accepted: 6 June 2019

Published online: 03 July 2019

\section{References}

1. von Strauss, E., Viitanen, M., De Ronchi, D., Winblad, B. \& Fratiglioni, L. Aging and the occurrence of dementia. Arch. Neurol. 56, 587-592 (1999).

2. Bennett, D. A. et al. Prevalence of Parkinsonian signs and associated mortality in a community population of older people. N. Engl. J. Med. 334, 71-76 (1996).

3. Fjell, A. M. \& Walhovd, K. B. Structural brain changes in aging: courses, causes and cognitive consequences. Rev. Neurosci. 21, 187-221 (2010).
4. Seeman, M. V. Psychopathology in women and men: focus on female hormones. Am. J. Psychiatry 154, 1641-1647 (1997).

5. Jessen, H. M. \& Auger, A. P. Sex differences in epigenetic mechanisms may underlie risk and resilience for mental health disorders. Epigenetics 6, 857-861 (2011).

6. Lu, T. et al. Gene regulation and DNA damage in the ageing human brain. Nature 429, 883-891 (2004)

7. Shi, L., Zhang, Z. \& Su, B. Sex biased gene expression profiling of human brains at major developmental stages. Sci. Rep. 6, 1-9 (2016).

8. Trabzuni, D. et al. Widespread sex differences in gene expression and splicing in the adult human brain. Nat. Commun. 4, 1-7 (2013).

9. Viñuela, A. et al. Age-dependent changes in mean and variance of gene expression across tissues in a twin cohort. Hum. Mol. Genet. 27, 732-741 (2018).

10. Bale, T. L. \& Epperson, C. N. Sex differences and stress across the lifespan. Nat. Neurosci. 18, 1413-1420 (2015).

11. Mostafavi, S. et al. A molecular network of the aging human brain provides insights into the pathology and cognitive decline of Alzheimer's disease. Nat. Neurosci. 21, 811-819 (2018).

12. Krishnan, V. et al. Histone H4 lysine 16 hypoacetylation is associated with defective DNA repair and premature senescence in Zmpste24-deficient mice. Proc. Natl Acad. Sci. USA 108, 12325-12330 (2011).

13. Nativio, R. et al. Dysregulation of the epigenetic landscape of normal aging in Alzheimer's disease. Nat. Neurosci. 21, 497-505 (2018).

14. Singh, P. \& Thakur, M. K. Reduced recognition memory is correlated with decrease in DNA methyltransferase1 and increase in histone deacetylase2 protein expression in old male mice. Biogerontology 15, 339-346 (2014).

15. Chouliaras, L. et al. Histone deacetylase 2 in the mouse hippocampus: attenuation of age-related increase by caloric restriction. Curr. Alzheimer Res. 10, 868-876 (2013).

16. dos Santos Sant' Anna, G. et al. Histone deacetylase activity is altered in brain areas from aged rats. Neurosci. Lett. 556, 152-154 (2013).

17. Peleg, S. et al. Altered histone acetylation is associated with age-dependent memory impairment in mice. Science 328, 753-756 (2010).

18. Benito, E. et al. HDAC inhibitor-dependent transcriptome and memory reinstatement in cognitive decline models. J. Clin. Invest. 125, 3572-3584 (2015).

19. Singh, P. \& Thakur, M. K. Histone Deacetylase 2 Inhibition Attenuates Downregulation of Hippocampal Plasticity Gene Expression during Aging Mol. Neurobiol. 1-11, https://doi.org/10.1007/s12035-017-0490-x (2017).

20. Gräff, J. et al. An epigenetic blockade of cognitive functions in the neurodegenerating brain. Nature 483, 222-226 (2012).

21. Guan, J. S. et al. HDAC2 negatively regulates memory formation and synaptic plasticity. Nature 459, 55-60 (2009).

22. Burke, S. N. \& Barnes, C. A. Neural plasticity in the ageing brain. Nat. Rev. Neurosci. 7, 30-40 (2006).

23. Wey, H. Y. et al. Insights into neuroepigenetics through human histone deacetylase PET imaging. Sci. Transl. Med. 8, pp. 351 ral06 https://doi.org/ 10.1126/scitranslmed.aaf7551 (2016)

24. Shen, S. et al. Age-dependent epigenetic control of differentiation inhibitors is critical for remyelination efficiency. Nat. Neurosci. 11, 1024-1034 (2008).

25. Baltan, S., Murphy, S. P., Danilov, C. A., Bachleda, A. \& Morrison, R. S. Histone deacetylase inhibitors preserve white matter structure and function during ischemia by conserving atp and reducing excitotoxicity. J. Neurosci. 31, 3990-3999 (2011).

26. Brügger, V. et al. Delaying histone deacetylase response to injury accelerates conversion into repair Schwann cells and nerve regeneration. Nat. Commun. 8, 14272 (2017).

27. Ge, Z. et al. Vorinostat, a histone deacetylase inhibitor, suppresses dendritic cell function and ameliorates experimental autoimmune encephalomyelitis. Exp. Neurol. 241, 56-66 (2013).

28. Tsai, H.-W., Grant, P. A. \& Rissman, E. F. Sex differences in histone modifications in the neonatal mouse brain. Epigenetics 4, 47-53 (2009).

29. Zhao, Z., Fan, L. \& Frick, K. M. Epigenetic alterations regulate estradiolinduced enhancement of memory consolidation. Proc. Natl Acad. Sci. USA 107, 5605-5610 (2010).

30. Matsuda, K. I. et al. Histone deacetylation during brain development is essential for permanent masculinization of sexual behavior. Endocrinology 152, 2760-2767 (2011).

31. Jessen, H. M., Kolodkin, M. H., Bychowski, M. E., Auger, C. J. \& Auger, A. P. The nuclear receptor corepressor has organizational effects within the developing amygdala on juvenile social play and anxiety-like behavior. Endocrinology 151, 1212-1220 (2010).

32. Thevenet, L. et al. Regulation of human SRY subcellular distribution by its acetylation/deacetylation. EMBO J. 23, 3336-3345 (2004).

33. Jeppesen, P. \& Turner, B. M. The inactive X chromosome in female mammals is distinguished by a lack of histone $\mathrm{H} 4$ acetylation, a cytogenetic marker for gene expression. Cell 74, 281-289 (1993). 
34. de rubertis, F. et al. The histone deacetylase RPD3 conteracts genomic silencing in Drosophila and yeast. Nature 384, 589-591 (1997).

35. Wang, C. et al. In vivo imaging of histone deacetylases (HDACs) in the central nervous system and major peripheral organs. J. Med. Chem. 57, 7999-8009 (2014).

36. Schroeder, F. A. et al. PET imaging demonstrates histone deacetylase target engagement and clarifies brain penetrance of known and novel small molecule inhibitors in rat. ACS Chem. Neurosci. 10, 1055-1062 (2014).

37. Wey, H.-Y. et al. Kinetic analysis and quantification of $\left[{ }^{11} \mathrm{C}\right]$ Martinostat for in vivo HDAC imaging of the brain. ACS Chem. Neurosci. 6, 708-715 (2015).

38. Gilbert, T. M. et al. PET neuroimaging reveals histone deacetylase dysregulation in schizophrenia. J. Clin. Invest. 129, 364-372 (2019).

39. Loggia, M. L. et al. Evidence for brain glial activation in chronic pain patients. Brain 138, 604-615 (2015)

40. Zürcher, N. R. et al. Increased in vivo glial activation in patients with amyotrophic lateral sclerosis: assessed with $\left[{ }^{11} \mathrm{C}\right]-\mathrm{PBR} 28$. Neuroimage Clin. 7 , 409-414 (2015).

41. Scahill, R. I. et al. A longitudinal study of brain volume changes in normal aging using serial registered magnetic resonance imaging. Arch. Neurol. 60 989 (2003).

42. Castellano, J. F. et al. Age-related memory impairment is associated with disrupted multivariate epigenetic coordination in the hippocampus. PLoS. ONE. 7, e33249 (2012).

43. Pasternak, O., Kelly, S., Sydnor, V. J. \& Shenton, M. E. Advances in microstructural diffusion neuroimaging for psychiatric disorders. Neuroimage 182, 259-282 (2018).

44. Fritzsche, K. H., Laun, F. B., Meinzer, H. P. \& Stieltjes, B. Opportunities and pitfalls in the quantification of fiber integrity: what can we gain from Q-ball imaging? Neuroimage 51, 242-251 (2010).

45. Gorczewski, K., Mang, S. \& Klose, U. Reproducibility and consistency of evaluation techniques for HARDI data. Magn. Reson. Mater. Phys., Biol. Med. 22, 63-70 (2009).

46. Lindemer, E. R., Greve, D. N., Fischl, B. R., Augustinack, J. C. \& Salat, D. H. Regional staging of white matter signal abnormalities in aging and Alzheimer's disease. Neuroimage Clin. 14, 156-165 (2017).

47. He, Y. et al. The transcription factor Yin Yang 1 is essential for oligodendrocyte progenitor differentiation. Neuron 55, 217-230 (2007).

48. Jacob, C. et al. HDAC1 and HDAC2 control the transcriptional program of myelination and the survival of Schwann cells. Nat. Neurosci. 14, 429-436 (2011).

49. Ye, F. et al. HDAC1 and HDAC2 regulate oligodendrocyte differentiation by disrupting the B-catenin-TCF interaction. Nat. Neurosci. 12, 829-838 (2009).

50. Mayer, J. D., Salovey, P., Caruso, D. R. \& Sitarenios, G. Measuring emotional intelligence with the MSCEIT V2.0. Emotion 3, 97-105 (2003).

51. Wang, Y., Metoki, A., Alm, K. H. \& Olson, I. R. White matter pathways and social cognition. Neurosci. Biobehav. Rev. 90, 350-370 (2018).

52. Wang, Y. \& Olson, I. R. The original social network: white matter and social cognition. Trends Cogn. Sci. 22, 504-516 (2018).

53. Covington, H. E., Vialou, V. F., LaPlant, Q., Ohnishi, Y. N. \& Nestler, E. J. Hippocampal-dependent antidepressant-like activity of histone deacetylase inhibition. Neurosci. Lett. 493, 122-126 (2011).

54. Schroeder, F. A., Lin, C. L., Crusio, W. E. \& Akbarian, S. Antidepressant-like effects of the histone deacetylase inhibitor, sodium butyrate, in the mouse. Biol. Psychiatry 62, 55-64 (2007).

55. Tysnes, O. B. \& Storstein, A. Epidemiology of Parkinson's disease. J. Neural Transm. 124, 901-905 (2017).

56. Chen, Y. et al. HDAC-mediated deacetylation of NF- $\kappa B$ is critical for Schwann cell myelination. Nat. Neurosci. 14, 437-441 (2011).

57. $\mathrm{Lu}, \mathrm{T}$. et al. REST and stress resistance in ageing and Alzheimer's disease. Nature 507, 448-454 (2014).

58. Gjoneska, E. et al. Conserved epigenomic signals in mice and humans reveal immune basis of Alzheimer's disease. Nature 518, 365-369 (2015).

59. Sewal, A. S. et al. Experience modulates the effects of histone deacetylase inhibitors on gene and protein expression in the hippocampus: impaired plasticity in aging. J. Neurosci. 35, 11729-11742 (2015).

60. Cosgrove, K. P., Mazure, C. M. \& Staley, J. K. Evolving knowledge of sex differences in brain structure, function, and chemistry. Biol. Psychiatry 62, 847-855 (2007).

61. Parsey, R. V. et al. Effects of sex, age, and aggressive traits in man on brain serotonin 5-HT1A receptor binding potential measured by PET using [C-11] WAY-100635. Brain Res. 954, 173-182 (2002).

62. Kaufman, J. et al. Quantification of the serotonin 1A receptor using PET: identification of a potential biomarker of major depression in males. Neuropsychopharmacology 40, 1692-1699 (2015).

63. Souslova, T., Mirédin, K., Millar, A. M. \& Albert, P. R. Recruitment by the repressor Freud-1 of histone deacetylase-brg1 chromatin remodeling complexes to strengthen HTR1A gene repression. Mol. Neurobiol. 54, 8263-8277 (2017).
64. Kurita, M. et al. HDAC2 regulates atypical antipsychotic responses through the modulation of mGlu2 promoter activity. Nat. Neurosci. 15, 1245-1254 (2012).

65. Ibi, D. et al. Antipsychotic-induced Hdac2 transcription via NF-kB leads to synaptic and cognitive side effects. Nat. Neurosci. 20, 1247-1259 (2017).

66. Schroeder, F. A. et al. A selective HDAC $1 / 2$ inhibitor modulates chromatin and gene expression in brain and alters mouse behavior in two mood-related tests. PLoS. ONE. 8, e71323 (2013).

67. Covington, H. E. et al. Antidepressant actions of histone deacetylase inhibitors. J. Neurosci. 29, 11451-11460 (2009).

68. Gräff, J. et al. Epigenetic priming of memory updating during reconsolidation to attenuate remote fear memories. Cell 156, 261-276 (2014).

69. Fischer, A., Sananbenesi, F., Wang, X., Dobbin, M. \& Tsai, L. H. Recovery of learning and memory is associated with chromatin remodelling. Nature 447, 178-182 (2007).

70. Kilgore, M. et al. Inhibitors of class 1 histone deacetylases reverse contextual memory deficits in a mouse model of alzheimer's disease. Neuropsychopharmacology 35, 870-880 (2010).

71. Strebl, M. G. et al. HDAC6 brain mapping with [18f]bavarostat enabled by a Ru-mediated deoxyfluorination. ACS Cent. Sci. 3, 1006-1014 (2017)

72. Jenkinson, M., Bannister, P., Brady, M. \& Smith, S. Improved optimization for the robust and accurate linear registration and motion correction of brain images. Neuroimage 17, 825-841 (2002)

73. Smith, S. M. et al. Advances in functional and structural MR image analysis and implementation as FSL. Neuroimage 23, 208-219 (2004).

74. Andersson, J. L. R., Jenkinson, M. \& Smith, S. Non-linear registration, aka spatial normalisation. FMRIB Technial Report TR07JA2. (Oxford University, Oxford, 2007)

75. Iglesias, J. E. et al. Bayesian segmentation of brainstem structures in MRI. Neuroimage 113, 184-195 (2015).

76. Winkler, A. M., Ridgway, G. R., Webster, M. A., Smith, S. M. \& Nichols, T. E. Permutation inference for the general linear model. Neuroimage 92, 381-397 (2014).

77. Beckmann, C. F. et al. Applying FSL to the FIAC data: Model-based and model-free analysis of voice and sentence repetition priming. Hum. Brain. Mapp. 27, 380-391 (2006).

78. Fischl, B. et al. Whole brain segmentation: automated labeling of neuroanatomical structures in the human brain. Neuron 33, 341-355 (2002)

79. Greve, D. N. et al. Different partial volume correction methods lead to different conclusions: an 18 F-FDG-PET study of aging. Neuroimage 132, 334-343 (2016).

80. Tzourio-Mazoyer, N. et al. Automated anatomical labeling of activations in SPM using a macroscopic anatomical parcellation of the MNI MRI singlesubject brain. Neuroimage 15, 273-289 (2002).

\section{Acknowledgements}

We thank B. Dickerson, A. Kendall, M. Wentworth, O. Johnson-Akeju, M. Albers, and N. Nortelus for consenting subjects and/or medical coverage; E. Pierce and O. Johnson Akeju for arterial-line placement; J. Sore and the radiopharmacy team for radiotracer synthesis; G. Arabasz, S. Hsu, and R. Butterfield for assistance with MR-PET imaging; and A. Zhu for imaging data collection. We are grateful to C. Catana, D. Izquierdo, and D. Chonde for PET image analysis advice and A. Harikumar for advice on cognitive metrics. We thank M. Loggia, D. Albrecht, and M. Riley for helpful comments. This research received funding from the National Institute on Drug Abuse (R01DA030321 to J.M.H. and R00DA037928 to H-Y.W.), National Institute of Mental Health (R21 MH11197101A1 to J.M.H., R01 MH102377 and K24 MH110807 to M.K., R03 MH110745 to A.E.L., and R01MH108574 to O.P.), Harvard/MGH Nuclear Medicine Training Program from the Department of Energy (DE-SC0008430 to H-Y.W., T.M.G., and C.W.), Brain and Behavior Foundation Independent Investigator Award (J.M.H.) and Young Investigator Award (T.M.G.), American Federation for Aging Research (AFAR) New Investigator Award in Alzheimer's Disease (H-Y.W.), MGH Research Scholar's Program (J.M.H.), MGH ECOR Fund for Medical Discovery (T.M.G.), and Athinoula A. Martinos Center Postdoctoral Pilot Funding (T.M.G.). This research was carried out at the Athinoula A. Martinos Center for Biomedical Imaging at MGH, using resources provided by the Center for Functional Neuroimaging Technologies, P41EB015896, a P41 Biotechnology Resource Grant supported by the National Institute of Biomedical Imaging and Bioengineering (NIBIB), and the Neuroimaging Analysis Center, P41EB015902, a P41 supported by NIBIB. This work also involved the use of instrumentation supported by the National Institutes of Health (NIH) Shared Instrumentation Grant Program; specifically, S10RR017208-01A1, S10RR026666, S10RR022976, S10RR019933, S10RR023043, and S10RR023401. Human tissue was obtained from the NIH Neurobiobank at the University of Maryland, Baltimore, MD. Tissue was also obtained through the Harvard Brain Tissue Resource Center, funded through the NIH-NeuroBiobank HHSN-271-2013-00030C [The National Institute of Mental Health (NIMH), National Institute of Neurological Diseases and Stroke (NINDS), 
and Eunice Kennedy Shriver National Institute of Child Health and Human Development (NICHD)].

\section{Author contributions}

T.M.G., N.R.Z., M.C.C. and J.M.H. designed the study. T.M.G., N.R.Z., B.G.H., A.J.P., A.B., C.J.W., M.L.H., M.K., S.M.S., U.M., F.A.S., C.W., J.L.R., D.J.H. and H.Y.-W. collected human imaging data, demographic data and/or cognitive data. T.M.G., N.R.Z., C.-E.J.T., M.D., A.E.L., D.N.G., O.P., M.K. and H.-Y.W. analyzed human imaging data. M.C.C. collected and analyzed biochemical data. T.M.G., N.R.Z., M.C.C., C.-E.J.T. and B.G.H. performed statistical analyses. T.M.G., N.R.Z., M.C.C. and J.M.H. wrote the paper. All authors edited the paper.

\section{Additional information}

Supplementary Information accompanies this paper at https://doi.org/10.1038/s41467019-11031-0.

Competing interests: The content is solely the responsibility of the authors and does not necessarily represent the official views of Massachusetts General Hospital, Harvard University, and its affiliated academic healthcare centers, or the NIH. Intellectual property (IP) has been filed around $\left[{ }^{11} \mathrm{C}\right]$ Martinostat by J.M.H., C.W. and F.A.S. A portion of this IP has been licensed. J.M.H. is a co-founder of Eikonizo Therapeutics, Inc. and consults for Psy Therapeutics, Inc. F.A.S. is a co-founder and current employee of Eikonizo Therapeutics, Inc. T.M.G. is a current employee of Eikonizo Therapeutics, Inc. The remaining authors declare no competing interests.
Reprints and permission information is available online at http://npg.nature.com/ reprintsandpermissions/

Peer review information: Nature Communications thanks the anonymous reviewer(s) for their contribution to the peer review of this work.

Publisher's note: Springer Nature remains neutral with regard to jurisdictional claims in published maps and institutional affiliations.

(c) (i) Open Access This article is licensed under a Creative Commons Attribution 4.0 International License, which permits use, sharing, adaptation, distribution and reproduction in any medium or format, as long as you give appropriate credit to the original author(s) and the source, provide a link to the Creative Commons license, and indicate if changes were made. The images or other third party material in this article are included in the article's Creative Commons license, unless indicated otherwise in a credit line to the material. If material is not included in the article's Creative Commons license and your intended use is not permitted by statutory regulation or exceeds the permitted use, you will need to obtain permission directly from the copyright holder. To view a copy of this license, visit http://creativecommons.org/ licenses/by/4.0/.

(C) The Author(s) 2019 